\title{
Analyse et Interprétation des Données Magnétiques au Sol du Secteur NNE d'Achemmach (Maroc central)
}

\author{
Mezougane Hafid, \\ Aissa Mohamed, \\ Moussaid Azizi, \\ Souiri Muhammad,
}

Laboratoire d'Etudes des Ressources Minérales et Energétiques, Département de Géologie, Faculté des Sciences, Zitoune, Meknès, Maroc

Souiah Mohamed, Managem/ONA. Center, Tower A, Mâarif Casablanca, Morocco

Doi:10.19044/esj.2019.v15n30p439 URL:http://dx.doi.org/10.19044/esj.2019.v15n30p439

\section{Résumé}

La région NNE d'Achemmach se situe à $40 \mathrm{~km}$ environ au SSW de la ville de Meknès et à $6 \mathrm{~km}$ au NE de la mine d'El Hammam. Elle fait partie du massif paléozoïque du Maroc central. Géologiquement, le secteur est formé de deux ensembles paléozoïques: (i) Un ensemble méta-sédimentaire composé de 2 unités : l'une calcaire et shisto-gréseuse d'âge Viséen moyensupérieur et l'autre flyschoïde d'âge Viséen supérieur-Namurien, et (ii) un ensemble magmatique matérialisé par des roches volcaniques (basaltes en coussins), des roches hypo-volcaniques (dolérites) et des roches plutoniques (gabbros à olivine). Structuralement, les terrains du Viséen-Namurien de la région NNE d'Achemmach sont affectés par l'évènement majeur namurowestphalien de l'orogenèse hercynienne (raccourcissement NW-SE). Celui ci est responsable d'un plissement synchisteux d'orientation générale NE-SW à NNE-SSW, de cisaillements NE-SW d'ampleur régionale, du développement de failles NE-SW à NNE-SSW subparallèles à la schistosité et de failles N130 à N150 à jeu senestre. Une campagne géophysique par magnétisme du champ total a été réalisée sur ce secteur. Le recoupement des données de cette campagne avec les données géologiques montre: i) une anomalie bien marquée à l'ouest du secteur, qui coïncide avec l'affleurement des roches basiques et des pélites verdâtres encaissantes. Celle-ci pourrait être originaire soit d'une richesse des roches basiques (gabbros, laves basiques et les dolérites)en minéraux magnétiques (magnétite),soit d'une différence de susceptibilité magnétique entre les différents faciès, soit encore d'un corps magnétique 
perturbateur non affleurant à la surface ; et ii) une anomalie à l'est, de moindre extension, et qui coïncide globalement avec la zone de cisaillement NE-SW mettant en contact les flyschs du Viséen supérieur-Namurien à l'est et la série calcaire et gréso-pélitique à volcanisme du Viséen moyen-supérieur à l'ouest. Cet axe anomalique pourait être dû à une grande différence de susceptibilité magnétique entre les formations en contact. Cependant l'expression de l'anomalie uniquement dans la partie sud de la zone de cisaillement mais pas dans sa partie nord, fait penser à une source autre que la différence de susceptibilité magnétique telle qu'une concentration de minéraux magnétiques (pyrrhotite, magnétite...).

Mots clés: Maroc Central, NNE d'Achemmach, Anomalies magnétiques, Minéraux magnétiques

\title{
Analysis and Interpretation of Ground-Based Magnetic Data of the NNE Part of Achemmach Mining Field (Central Morocco)
}

\author{
Mezougane Hafid, \\ Aissa Mohamed, \\ Moussaid Azizi, \\ Souiri Muhammad,
}

Laboratoire d'Etudes des Ressources Minérales et Energétiques, Département de Géologie, Faculté des Sciences, Zitoune, Meknès, Maroc

Souiah Mohamed, Managem/ONA. Center, Tower A, Mâarif Casablanca, Morocco

\begin{abstract}
The Achemmach mining field is about $40 \mathrm{~km}$ from the SSW of the city of Meknes and $6 \mathrm{~km}$ NE of the El Hammam mine. Our study area is located in the NNE of this mining zone. It is in the Paleozoic massif of the Central Morocco. Geologically, the area is made up of two Paleozoic groups: (i) A metasedimentary set which is composed of two units: limestone and shallysandstone of the Upper Middle ages and the other flyshoid of the Upper Visean-Namurian ages, and (ii) a magmatic set that ismaterialized by volcanic rocks (pillow basalt), hypo-volcanic rocks (dolerite) and plutonicrocks (olivine gabbros). Structurally, the Visean-Namurian formations of the NNE region of Achemmach are affected by the major Namuro-Westphalian event
\end{abstract}


of hercynian orogenesis, characterized by NW-SE shortening. This event is responsible for a general NE-SW to NNE-SSW folding, NE-SW shearat regional-scale, development of NE-SW to NNE-SSW subparallel to schistosity and N130 to N150sinistral faults. A geophysical survey by groundbased magnetism of the total field was carried out on this area. Crossreferencing of geophysical and geological data showed: i) a well-defined anomaly to the west of the area, which coincides with the outcrop of basic rocks and surrounding greenish pelites. This could be either from a richness of basic rocks (gabbros, basic lava and dolerites) in magnetic minerals (magnetite),or from a difference in magnetic susceptibility between the different facies, or from a disturbing magnetic body not flush at the surface; and ii) an anomaly to the east, of lesser extent, which generally coincides with the NE-SW shear zone bringing together the flysches of the Upper ViseanNamurien to the east and the limestone and sandstone volcano series of the Middle Upper Visean to the west. This anomalous axis could be due to a large difference in magnetic susceptibility between superimposed formations. However, the expression of the anomaly only in the southern part of the shear zone but not in its northern part suggests a source other than the difference in magnetic susceptibilitysuch as a concentration of magnetic minerals (pyrrhotite, magnetite...).

Keywords: Central Morocco, NNE of Achemmach, Magnetic anomalies, Magnetic minerals

\section{Introduction}

De toutes les méthodes géophysiques utilisées en exploration minière, la prospection magnétique et surement la plus usuelle. Le succès de la grande «popularité» de son utilisation est attribué à son coût peu élevé. De plus c'est une méthode aux applications très variées allant de la cartographie géologique à la prospection de gisements. Qu'elle soit aéroportée ou au sol, la méthode magnétique comme toutes les méthodes géophysiques suit des principes physiques et requiert des corrections et des traitements appropriés après sa mise en œuvre.

La prospection magnétique est une méthode géophysique fondée sur l'interprétation des anomalies du champ magnétique terrestre décelant la présence dans le sous-sol de roches ou de substances plus ou moins aimantées comparées au champ magnétique total, qui est au Maroc de l'ordre de 40000 nanotesla. L'utilisation de cette méthode en prospection minière est réservée aux substances ferromagnétiques contenues dans les roches qui sont constitués en majeure partie de la magnétite ou de pyrrhotite.

Depuis la découverte en 1984 du gisement polymétallique de Hajjar dans le massif des Guemmassa (Felenc et al., 1989) à $\mathrm{Pb}, \mathrm{Zn}$, et $\mathrm{Cu}, 1$ 'attention 
des opérateurs miniers s'est orientée vers l'exploration des zones du massif hercynien à anomalies magnétiques.

C'est dans ce cadre que le présent travail a été réalisé. Il s'agit d'analyser et d'interpréter les données magnétiques du champ total à la lumière nouveaux résultats obtenus à partir de l'étude pétrographique et géochimique des formations magmatiques de ce secteur (Mezougane et al., 2019) dans le but d'apporter des précisions sur les sources potentielles, géologiques ou métalliques, qui peuvent être à l'origine des anomalies magnétiques

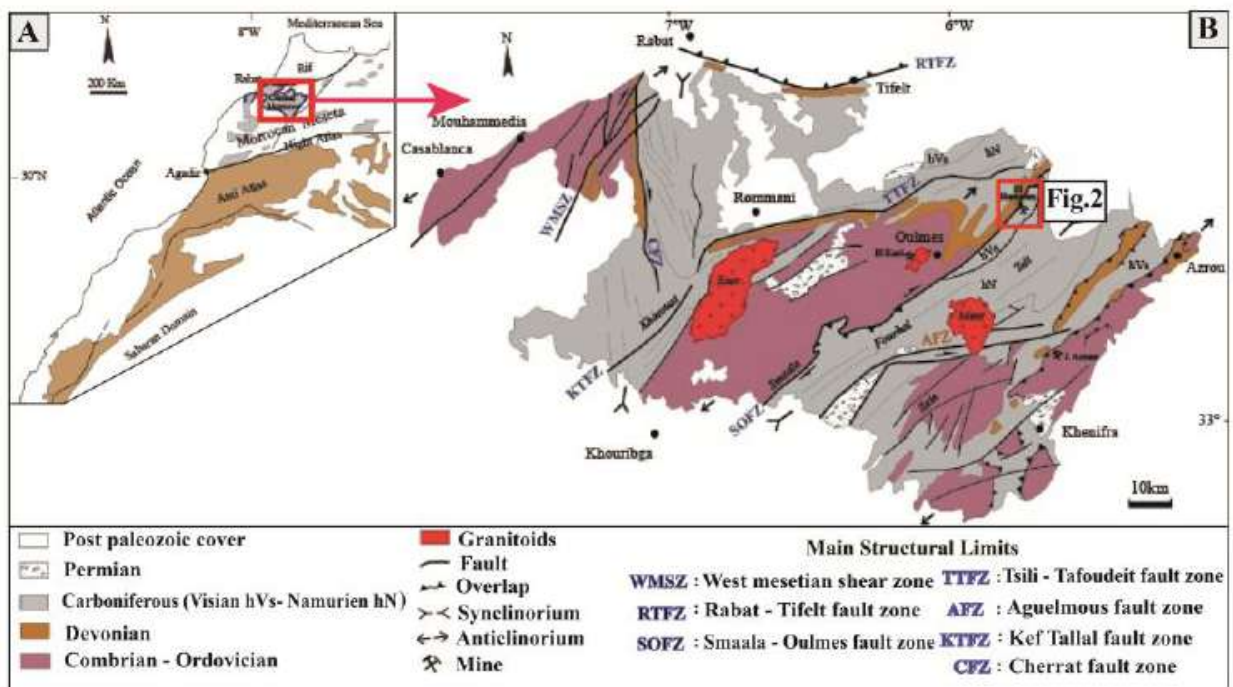

Figure 1: carte des domaines structuraux du Maroc avec localisation du Maroc central(A), (B): principales unités structurales (Michard 1976, Fadli et al. 1991).

\section{Cadre géologique}

Le secteur NNE d'Achemmach est situé à $40 \mathrm{~km}$ environ au SSW de la ville de Meknès et à $6 \mathrm{~km}$ au NE de la mine d'El Hammam. Il appartient à l'unité de Fourhal, située dans la partie NE du Massif Central Hercynien du Maroc (MCHM) (Fig. 1).Ce dernier, correspond à la zone septentrionale de la Meseta occidentale. Il est constitué d'un substratum néoprotérozoïque, d'affleurement réduit et local, représenté par des laves acides et intermédiaires (rhyolites et andésites) et de granites (Tahiri et al., 2010 ; Ouabid et al., 2017); d'un socle paléozoïque (du Cambrien au Permien) et d'une couverture mésocénozoïque . Les formations du Cambrien sont composées par des carbonates de plate-forme et des sédiments volcano-détritiques. L'Ordovicien est représenté par des dépôts détritiques (schistes, grès et quartzites) dans un environnement de type plate-forme marine peu profonde (Hamoumi, 1988). Le Silurien est caractérisé par une transgression glacio-eustatique donnant des pélites noires à graptolites et des carbonates. Le Dévonien (inférieur et moyen) est marqué par des dépôts argileux et carbonatés récifaux (Termier, 1936 ; 
Piqué \& Michard, 1981 ; Faik, 1988 ; Habibi, 1988 ; Bouabdelli, 1989 ; El Hassani, 1990 ; Fadli, 1990 ; Tahiri, 1991 ; Lazreq, 1983 ; Cattanéo et al.,1993 ; Zahraoui, 1994).

La déformation éovarisque (dévono-dinantienne) est responsable de la formation des bassins de Sidi Bettache et d'Azrou-Khénifra, d'abord en pullaparts contrôlés par des décrochements bordiers NNE-SSW à NE-SW, puis de type avant-pays contrôlés par des systèmes de plis et chevauchements vers l'WNW (Tahiri\&Hoepffner, 1987 ; Lakhloufi, 1988 ; Bouabdelli, 1989 ; Piqué \& Michard, 1989 ; El Hassani, 1990 ; Fadli, 1990 ; Izartet al.,2001 ; Ben Abbou et al., 2001 ; Berkhli et Vachard, 2002 ; Hoepffneret al.,2005). La sédimentation carbonifère est représentée surtout par les dépôts du Viséen et du Namurien. Le Viséen est constitué dans le Maroc central d'une épaisse série détritique et carbonatée (Termier, 1936 ; Allaryet al., 1972 ; Faik, 1988 ; Habibi, 1988 ; Bouabdelli, 1989 ; Ben Abbou, 1990 ; Piqué, 1994 ; Izartet al.,2001 ; BerkhlietVachard 2002), montrant des conglomérats, des schistes et des calcaires suivis par des formations gréso-pélitiques. Le Namurien est essentiellement composé de flyschs (Ben Abbou, 1990 ; Izart et al., 2001). A ces dépôts Viséens et Namuriens est associé un magmatisme sous forme de laves et d'intrusions basiques (basaltes, dolérites et gabbros) (Termier 1936, Sonnet 1981; Jebrak 1984; Kharbouch, 1994 ; Ben Abbou et al., 2001; Roddaz et al., 2002, Ntarmouchant, 2003).Ce magmatisme présente généralement une affinité alcaline transitionnelle à tholéiitique(Kharbouch, 1994) et localement (NE de la région du Fourhal) une tendance calco-alcaline (Roddaz et al., 2002).

Les dépôts du Permien inférieur (Autunien) sont représentés par des conglomérats, des grès, des siltites et des cinérites dans des bassins intracontinentaux, un important volcanisme calco-alcalin (trachytes, andésites et rhyolites) leur est associé (Cailleux, 1985 ; Zouine, 1986 ; El Wartiti, 1990 ; Remmal et al.,1999a ; Izartet al., 2001 ; Saidi et al., 2002 ; Saidi, 2005).

La déformation hercynienne majeure (Westphalien supérieurStéphanien), datée entre290 et 300Ma (Huon et al., 1987), et correspondant à une compression NW-SE, a structuré le massif central en unités anticlinoriales et synclinoriales, orientées NE-SW (Termier, 1936 ; Michard, 1976 ; Piqué \& Michard, 1981; Bouabdelli, 1989 ; Tahiri, 1994). Celles-ci sont séparées par des méga-failles dont celle de Smaala-Oulmès (ZFSO), laquelle seprolonge depuis les Smaala au sud (Cailleux, 1985 ; Oubbih, 1991 ; Tahiri\&Hoepffner, 1987) jusqu'au-delà du district d'El Hammam au nord (Agard,1966 ; Jebrak, 1984 ; Ben Abbou, 1990 ; Izartet al., 2001). Ces unités structurales montrent des plis synschisteux, déversés généralement vers le SE et localement vers le NW auxquels sont associés des chevauchements et des charriages (Tahiri, 1994). Dans ce domaine mesetien, les plutons granitiques syn à tardi tectoniques (Giuliani, 1989 ; Dahmani, 1985 ; Lagarde, 1985 ; Diot, 1989 ; 
Gasquet, 1996), sont calco-alcalins et pour la plupart peralumineux (Mriniet al., 1992 ; Boushaba\&Cailleux, 1992 ; Amenzou, 1997).

Le secteur d'étude, NNE d'Achmmach, se situe au NW du bassin Carbonifère de Fourhal (Fig.2). Il est limité du côté ouest par la ZCSO qui met en contact le bassin de Fourhal avec l'anticlinorium de Khouribga-Oulmès (Fig. 1).

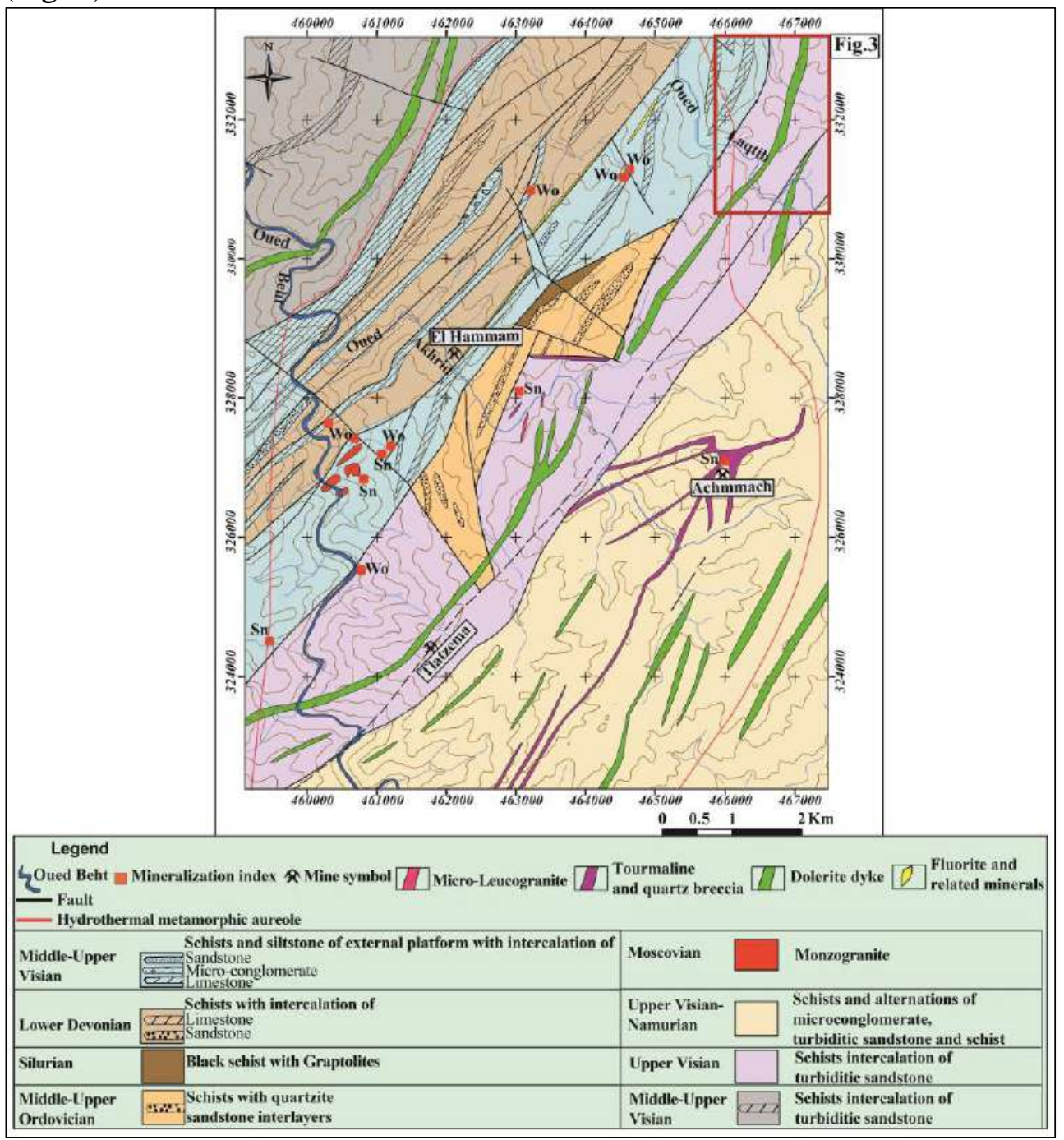

Figure 2: carte géologique du district d'Achmmach-El Hammam (Izart et al., 2001) montrant la localisation de la zone d'étude.

Géologiquement, le secteur est formé de deux ensembles paléozoïques qui constituent l'essentiel des terrains affleurant (Fig.3) :

1) Un ensemble méta-sédimentaires constitué par deux unités : i) une unité calcaire et shisto-gréseuse d'âge Viséen moyen à supérieur. Elle présente une direction globale NE-SW avec un pendage moyen de 60 à $70^{\circ}$ vers le $\mathrm{NW}$. 
Cette unité débute par des calcaires qui alternent avec des schistes et continue pardes schistes, des grès et des pélites verdâtres où s'intercale vers le nord un horizon décamétrique de laves en coussin. Vers le sud, la série se termine par les alternances de schistes et grès et par des schistes ardoisiers plus ou moins gréseux, et ii) une unité flyshoïde, par analogie avec les travaux de Ben Abbou, 1990, sur le Paléozoïque de la région d'Agourai, cette série est attribuée au Viséen supérieur-Namurien. Celle-ci repose sur la série schistogréso-volcanique supracalcaire (Viséen moyen-supérieur) par un contact anormal correspondant à la zone de cisaillement chevauchante NE-SW à NNE-SSW de Ticht Ougas (Fig.3). Cette formation débute par des microconglomérats granoclassés et se continue par des alternances rythmiques de pélites et grès, coiffées au sommet par une barre gréseuse. On ne connait pas de roches magmatiques associées à cette formation dans le secteur étudié.

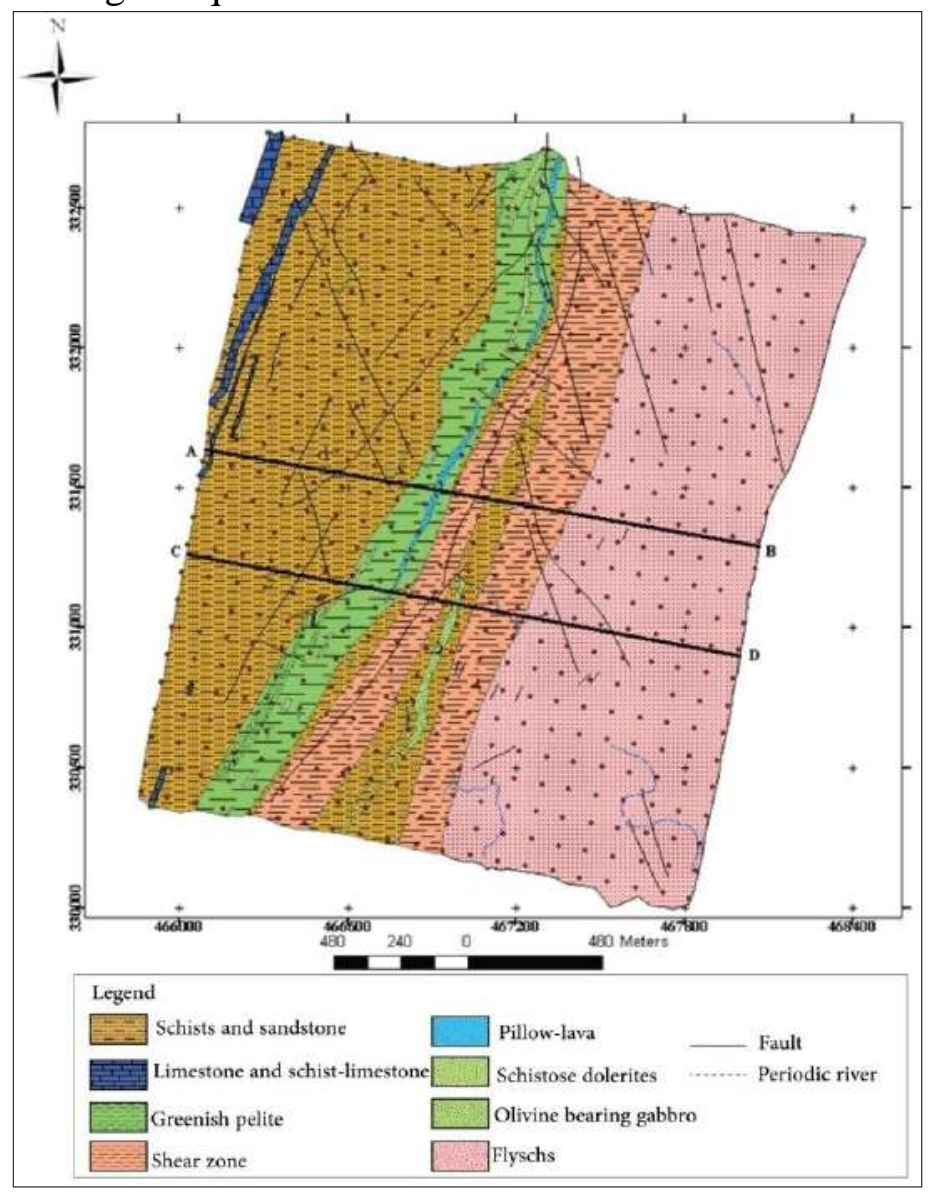

Figure 3 : carte géologique du secteur NNE Achemmach.

Malgré les effets de la tectonique hercynienne (succession de plis métriques, décamétriques et hectométriques), un log lithostratigraphique synthétique de cette série a été reconstitué(Fig.4). La série stratigraphique est 
composée d'une alternance rythmique de grès et de pélites épaisse de plusieurs centaines de mètres. La détermination de la puissance exacte de la série est rendue pratiquement impossible par l'intensité de la déformation hercynienne et par la couverture alluvionnaire quaternaire (Fig.4).

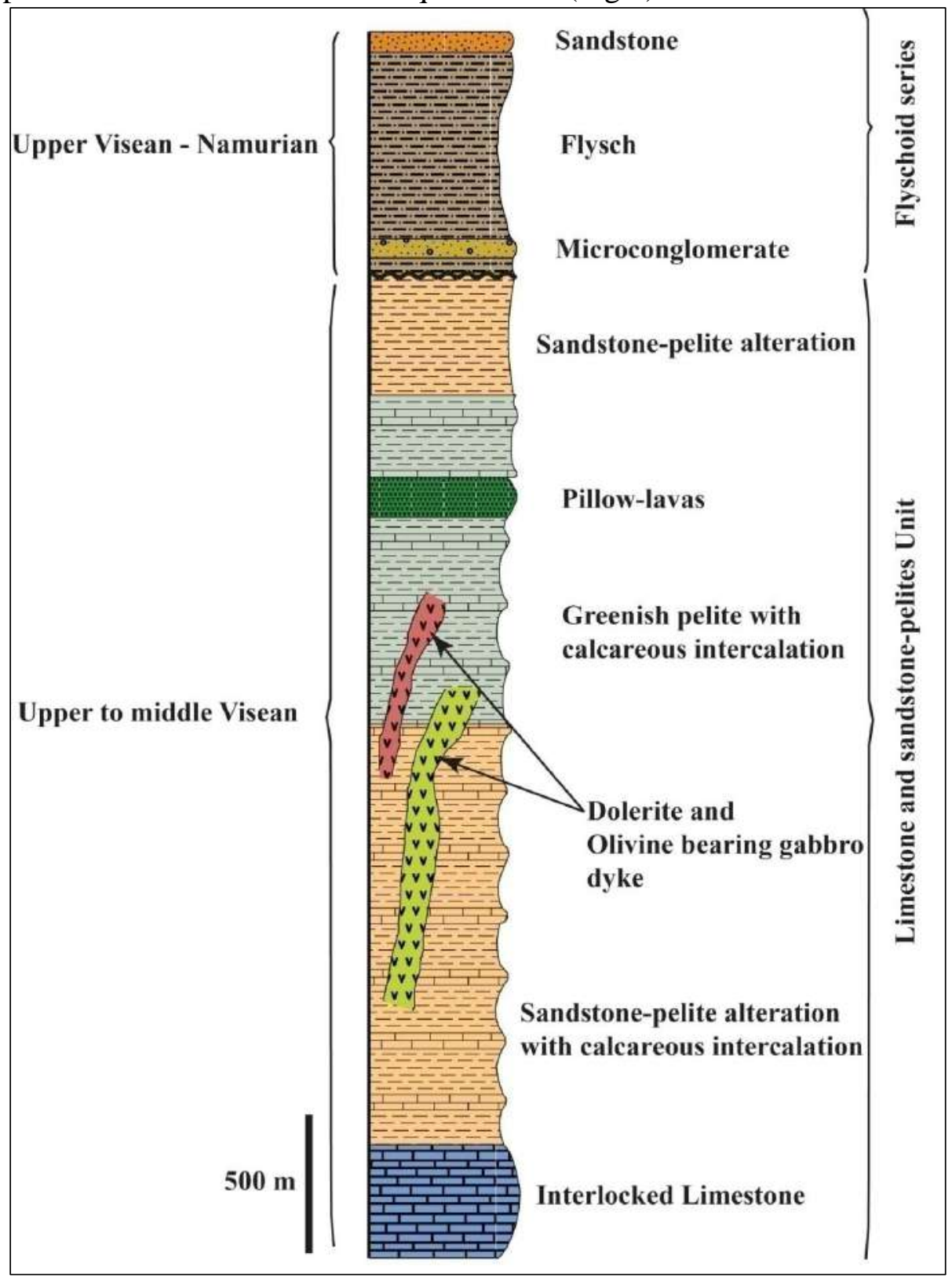

Figure 4 : log lithostratigraphique synthétique du secteur NNE d'Achemmach.

2) Un ensemble magmatique matérialisé par des roches volcaniques (basaltes en coussins), hypo-volcaniques (dolérites) et plutoniques (gabbros à olivine) 
(Fig.3). Ainsi, selon leur mode de mise en place, leur chronologie relative, leur pétrographie et leur mode de déformation, trois types peuvent en être distingués : i) les laves basiques (basaltes en coussins) de couleur verdâtre à vert sombre, affleurent à environ $150 \mathrm{~m}$ à l'ouest du secteur (Fig.3). Elles se présentent en un horizon continu, décamétrique (20 à $25 \mathrm{~m}$ ), de direction globale $\mathrm{N} 25^{\circ}$ à $\mathrm{N} 30^{\circ} \mathrm{E}$ et sont décalées par endroits par des failles transverses N130 à N165 $\mathrm{E}$ (Fig.3). Leur direction, globalement NE-SW au sud, devient N-S au nord du secteur. Elle est parallèle à celle de la stratification des pélites encaissantes. Leur pendage est vers le NW au sud et vers l'ouest au nord du secteur. Ces laves basiques sont encaissées dans un faciès de pélites verdâtres (Fig.3). A l'affleurement, elles sont facilement reconnaissables par leur pétrographie (texture et présence de vacuoles remplies de calcite) et la présence de coussins bien évidents. Cet horizon de laves est constitué par un empilement de coussins décimétriques à métriques, aux bordures figées, et montrant des fractures radiaires. Certaines bordures de coussins sont dessinées par de fines intercalations sédimentaires pélitiques (verdâtres) indiquant vraisemblablement une activité volcanique répétée en brefs épisodes. Ces laves montrent une hétérogénéité dans la distribution des vacuoles (résultant du dégazage de la lave) remplies de calcite tardive. On note aussi une variation latérale de la proportion et de la taille des vacuoles en allant du centre vers la bordure des coussins on trouve des niveaux très riches en vacuoles et d'autres qui en sont moins riches voire dépourvus. ii) Les dolérites déformées, métamorphisées et altérées, ces roches se présentent sous forme de dykes intrudant les schistes, les grés et les pélites de la série supra-calcaire d'âge viséen moyen - supérieur, elles présentent une direction globale NE - SW avec un pendage vers le nord- ouest .Leur puissance est très variable allant de moins de $0.5 \mathrm{~m}$ à plus de $15 \mathrm{~m}$. Deux critères permettent de qualifier ces corps magmatiques de dykes: leur direction globale n'est pas conforme avec la stratification. En effet, il y a toujours une différence de 5 à $10^{\circ}$ entre leur direction et celle de la stratification. L'effet thermique que développent ces dykes, lorsque leur puissance est importante, et qui se traduit par une silicification plus ou moins intense au niveau des contacts avec les schistes et les schisto-gréso-calcaires encaissant. Dans certains cas, on note que le mur de ces corps correspond à une brèche tectonique de 30 à $60 \mathrm{~cm}$, ce qui montre que leur mise en place est guidée par des failles précoces. La majorité de ces dolérites est affectée par une schistosité de flux très pénétrative, dont la direction globale est conforme avec celle des roches encaissantes (schistosité majeure). A l'affleurement, ces dolérites sont très altérées et dont la patine plus ou moins rougeâtre qui s'y développe est attribuée aux oxydes de fer en altération supergène. Et iii) les gabbros à olivine, qui affleurent en dykes de puissances variables, ils intrudent la série schisto-gréseuse supra-calcaire (Fig.3). Ces dykes ne sont pas affectés par la schistosité de la phase majeure et seraient donc postérieurs à cette phase. 
A la fin de la distension dévono-dinantienne et après le comblement du bassin viséen, le régime de contraintes jusqu'à présent distensif (NW-SE) a changé et devenu compressif annonçant l'orogenèse hercynienne.

Les terrains du Viséen moyen-supérieur et ceux du Viséen supérieurNamurien seront affectés par l'évènement majeur de l'orogenèse hercynienne. Il est responsable d'un plissement synchisteux sous un climat de métamorphisme épizonal (faciès des schistes verts à séricite-chlorite).

Cette orogenèse est marquée au début par un épisode majeur de déformation souple (plissement) suivi d'épisodes de déformation fragile (fracturation et développement de faille). L'étude structurale du secteur NNE d'Achemmach a montré une évolution comparable à celles des terrains du district d'EL Hammam et ceux de la région d'Agourai. On retiendra les points essentiels suivants :

- La structuration majeure NE-SW à NNE-SSW liée à la phase majeure de la tectonique hercynienne constitue l'empreinte du principal évènement tectonique régional. Elle se manifeste par un plissement synschisteux sous un climat métamorphique épizonal. Les plis sont métriques, décamétriques à hectométriques déversés vers le SW.

- Une importante activité magmatique volcanique et hypovolcanique précoce à la déformation majeure.

- Des zones de cisaillements d'ampleur régionale qui ont été chevauchantes avant d'être cisaillantes montrant un changement de direction en allant vers le Nord et marquant une virgation de la structure majeure.

- Des failles NE-SW à NNE-SSW subparallèles à la schistosité ayant parfois intensément affecté les terrains plissés.

- Des failles N130 à N150 vers l'Est ayant affecté toutes les structures précédentes et provoqué des décalages senestres.

Minéralisation

La minéralisation dans la région se divise en deux catégories : i) une minéralisation en dissémination dans les formations métasédimentaires, notamment les bancs métriques discontinus des grés quartzitiques et les roches volcaniques. Elle est constituée par des sulfures de fer (pyrite et pyrrhotite), de cuivre (chalcopyrite) et de plomb (galène) et ii) une minéralisation en veines dans les fractures affectant les formations de l'unité schisto-gréseuse ou en grains isolés dans le ciment de la brèche du conglomérat de base de la série flyschoïde. Elle est composée de, pyrite, pyrrhotite, arsénopyrite, cassitérite, stannite, chalcopyrite et des oxydes de fer (limonite et hématite). 


\section{Application de la méthode magnétique à la région NNE d'Achemmach}

\section{III.1 Appareils et méthode}

L'acquisition des données magnétiques a été faite à l'aide d'un magnétomètre à précision protonique modèle OMNI-Plus de la firme EDA Instruments. Ilconsiste en la mesure du champ géomagnétique total tous les $12,5 \mathrm{~m}$ le long des profils levés. La précision instrumentale des lectures est de l'ordre de 0,1 gamma.

Les corrections pour les variations diurnes du champ géomagnétique ont été appliquées soit automatiquement en utilisant un deuxième magnétomètre de type OMNI-Plus comme station de base, soit par la méthode des boucles. La station de base enregistre le champ magnétique total toutes les 20 secondes ;

Afin de faciliter la mise en œuvre du levé magnétique, les travaux ont commencé par l'implantation d'une grille dans le secteur étudié (NNE d'Achemmach). Cette grille est formée de trois réseaux de profils orientés N100 perpendiculairement à une ligne de base qui est orientée NNE-SSW. Les profils sont espacés dans le réseau Sud de $100 \mathrm{~m}$, dans le réseau médian de $200 \mathrm{~m}$ et dans le réseau nord de $400 \mathrm{~m}$. Les stations de mesure sont disposés le long de ces profils tous les 12,5 mètres (Fig.5).

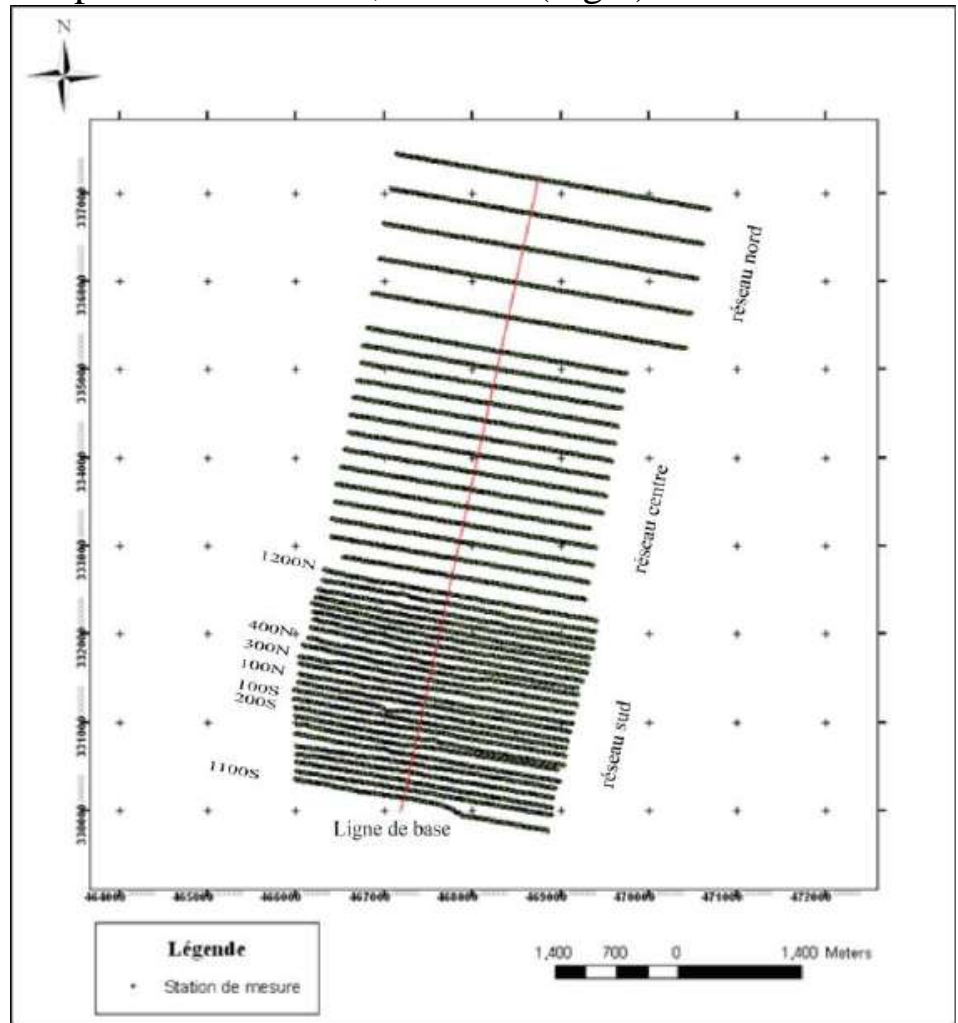

Figure 5 : grille du levé magnétique du secteur NNE d'Achemmach. 
Pour le traitement numérique et la transformation de la carte des données magnétique il est nécessaire d'avoir établi au préalable une grille régulière à partir des points de mesure.

Plusieurs essais ont été fait sur le logiciel Surfer tout en changeant la méthode d'interpolation à savoir ; Inverse Distance to a Power, Natural Neighbor, Minimum Curvature, la meilleure méthode prise est celle de Kriging. Le principe de cette dernière consiste à produire des cartes à partir des mesures acquises irrégulièrement espacées. Le «kriging» peut être un interpolateur exact ou sans heurt selon les paramètres personnalisés par l'utilisateur. Il incorpore l'anisotropie et les tendances fondamentales d'une façon efficace et normale.

En utilisant la méthode du kriging, on a établi une grille régulière à maille constante dont la longueur de $75 \mathrm{~m}$, est choisie en fonction de l'espacement entre les profils et l'équidistance des stations au sein du même profil. A partir de cette grille une carte en courbes du champ magnétique total a été établi (Fig. 6).

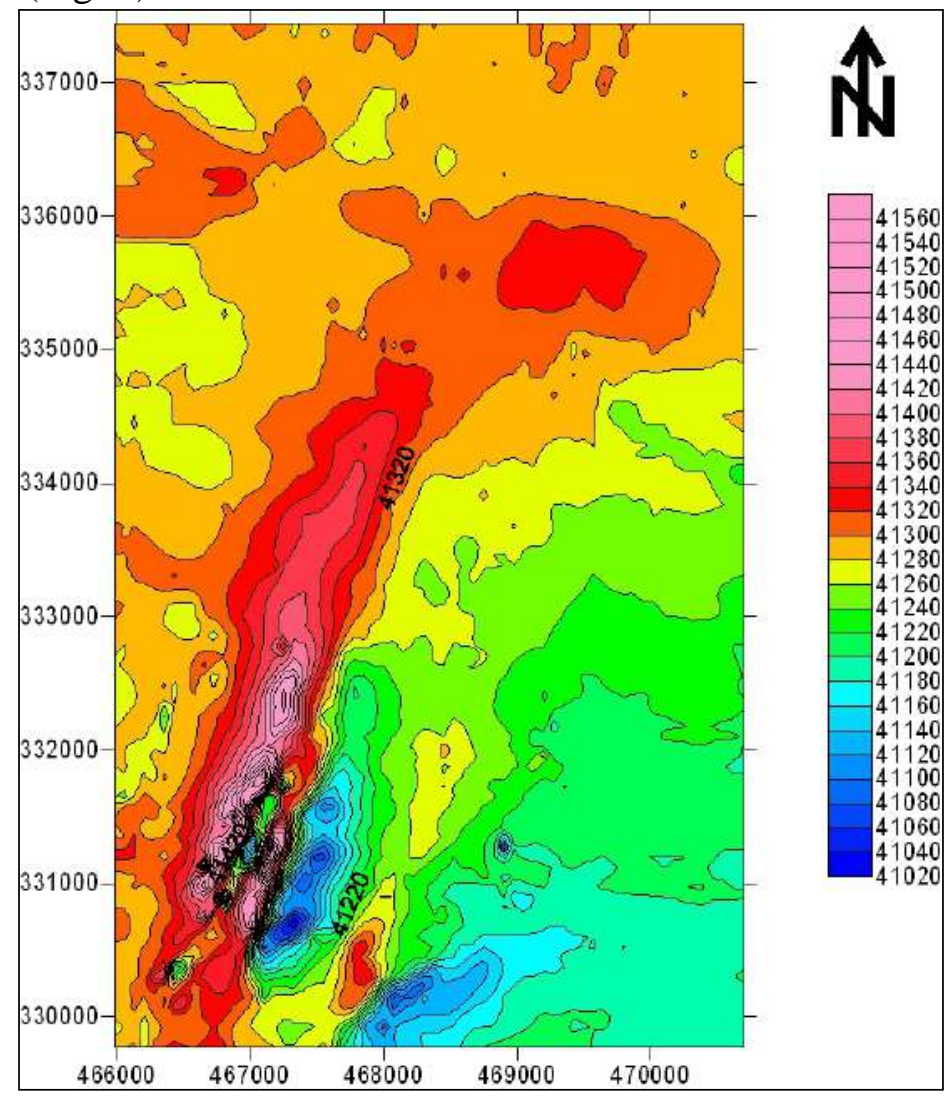

Figure 6 :carte des anomalies magnétiques du champ total (distance en $\mathrm{m}$ et valeur du champ en $\mathrm{nT}$ ). 


\section{III.2 Analyse des résultats}

L'interprétation d'une carte d'anomalies magnétiques repose sur plusieurs paramètres dont les plus importants sont :

$\rightarrow$ La qualité du levé magnétométrique, qui est étroitement liée à la précision et à la densité des points de mesure ;

$\Leftrightarrow$ Les transformations apportées aux anomalies, dont l'objectif est de montrer clairement les détails qui n'apparaissent pas sur la carte initiale ;

$\Leftrightarrow$ Toute autre information, d'ordre géologique, géophysique ou géochimique est précieuse afin de compléter et d'éclaircir les renseignements contenus dans la carte magnétique.

L'analyse de la carte magnétique élaborée fait ressortir deux zones principales (Fig. 7):

- Zone Nord située entre les coordonnées Y : 334000 m et Y : $337500 \mathrm{~m}$, où les valeurs du champ total sont comprises entre 41260 et $41360 \mathrm{nT}$.

- Zone Sud située entre les coordonnées Y : 329800 m et Y : $334000 \mathrm{~m}$. Cette zone est la plus perturbée magnétiquement et on y distingue quatre sous-zones magnétiques : ZMS1, ZMS2, ZMS3 et ZMS4. Ces sous zones sont toutes orientées environ $\mathrm{N} 25$.

Dans la zone Sud, d'ouest en est :

La zone ZM1 montre des valeurs qui sont relativement élevées mais homogènes (41240 à 41320 nT);

La zone ZM2 constitue le principal corridor anomalique. Elle est constituée de deux axes parallèles : l'un Est et l'autre Ouest, séparés par un léger creux magnétique. Les valeurs du champ total sont comprises entre 41220 et $41560 \mathrm{nT}$;

La zone ZM3 correspond probablement à la dépression magnétique associée à la zone ZM2. Les valeurs du champ total sont comprises entre 41020 et $41220 \mathrm{nT}$;

La zone ZM4 semble être le retour au niveau de la base magnétique.

Alors que dans la partie Nord, on observe la continuité du corridor magnétique (ZM2) dans la même direction N25. L'amplitude de l'anomalie devient plus faible en allant vers le Nord et sa largeur est en moyenne plus grande. On note aussi que l'extrémité nord du corridor marque une légère virgation vers l'est.

La zone ZM4 qui apparaît comme le retour au niveau de la base magnétique, dans la partie Sud, correspond en fait à un autre axe magnétique dans la partie Nord, en général plus faible mais de même orientation que la zone ZM2. 


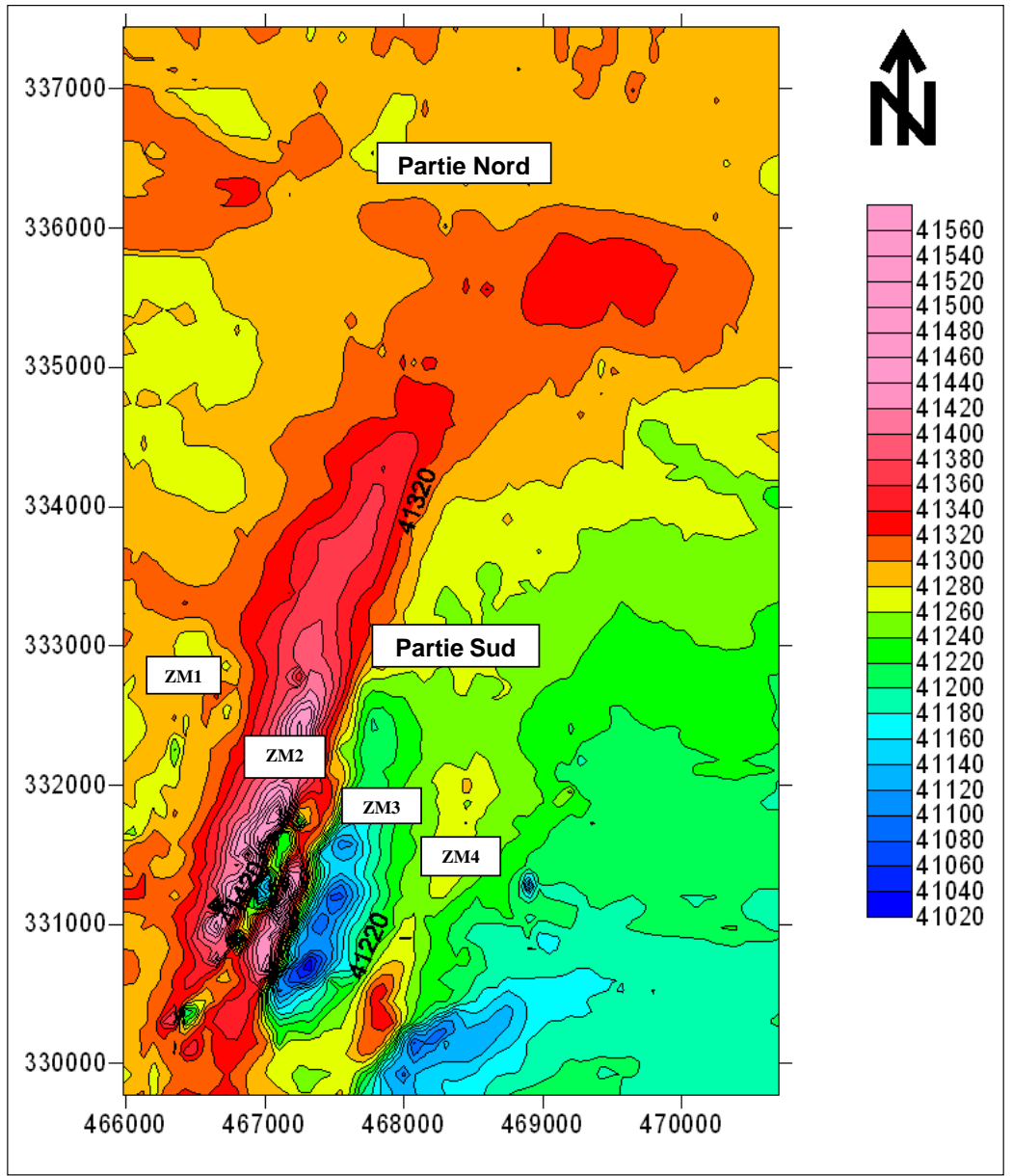

Figure 7 : carte des anomalies magnétiques du champ total (distance en $\mathrm{m}$ et valeur du champ en nT).

\section{III.3 Interprétation des anomalies magnétiques}

La superposition des données géologiques (faciès géologiques et accidents tectoniques du secteur étudié) et les anomalies magnétiques du champ total (Fig. 8), permet de dégager les remarques suivantes :

- Les deux principaux axes anomaliques de la zone ZMS2 ont la même direction que les unités géologiques (NE-SW);

- L'axe anomalique occidental coïncide avec l'affleurement des roches basiques et des pélites verdâtres encaissantes ;

- L'axe anomalique oriental de moindre extension, par rapport au premier axe anomalique, coïncide globalement avec la zone de cisaillement qui est orientée NE-SW mettant en contact les flyschs du Viséen supérieur-Namurien à l'Est et la série calcaire et grésopélitique à volcanisme du Viséen moyen-Supérieur à l'Ouest. 


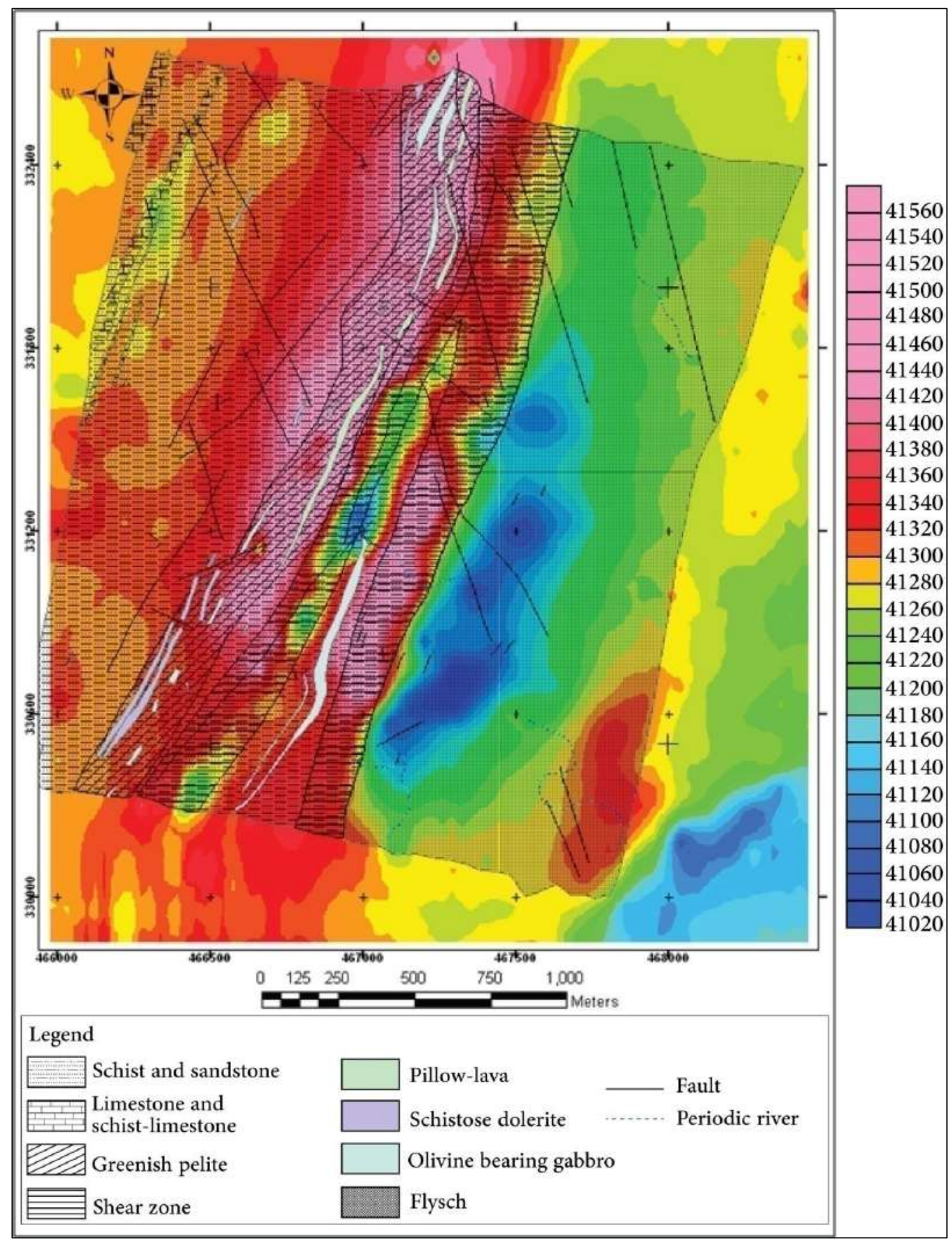

Figure 8 : carte de superposition du champ magnétique total avec la géologie du secteur.

Avant de proposer un essai d'interprétation de la présence des deux axes anomaliques de la zone ZM2 (Fig. 10), il convient de noter que les axes anomaliques sont plus ou moins rectilignes d'extension NE-SW. Cet état de 
fait suppose déjà que l'éventuel corps perturbateur ne peut se présenter qu'en un « corps » NE-SW de puissance largement inférieure à son extension.

1- L'axe anomalique occidental coïncide avec l'affleurement des roches basiques et des pélites verdâtres encaissantes tranchées vers l'Est par la zone de cisaillement occidental (Fig. 8). Plusieurs hypothèses pourraient être avancées pour expliquer la présence de cet axe anomalique:

- Sa liaison avec les roches volcaniques en pillow lava, mise en évidence à l'endroit du développement de l'axe anomalique. La magnitude bien marquée de cette anomalie suppose la richesse de ces laves en minéraux fortement magnétiques, tels que : la magnétite, ilménite et la pyrrhotite. Les observations macroscopiques et microscopiques montrent que ces laves sont parfois très riches en pyrrhotite disséminée dans les faciès fins (sans vacuoles) et concentrés autour des vacuoles à remplissage de calcite (Fig.9) ;

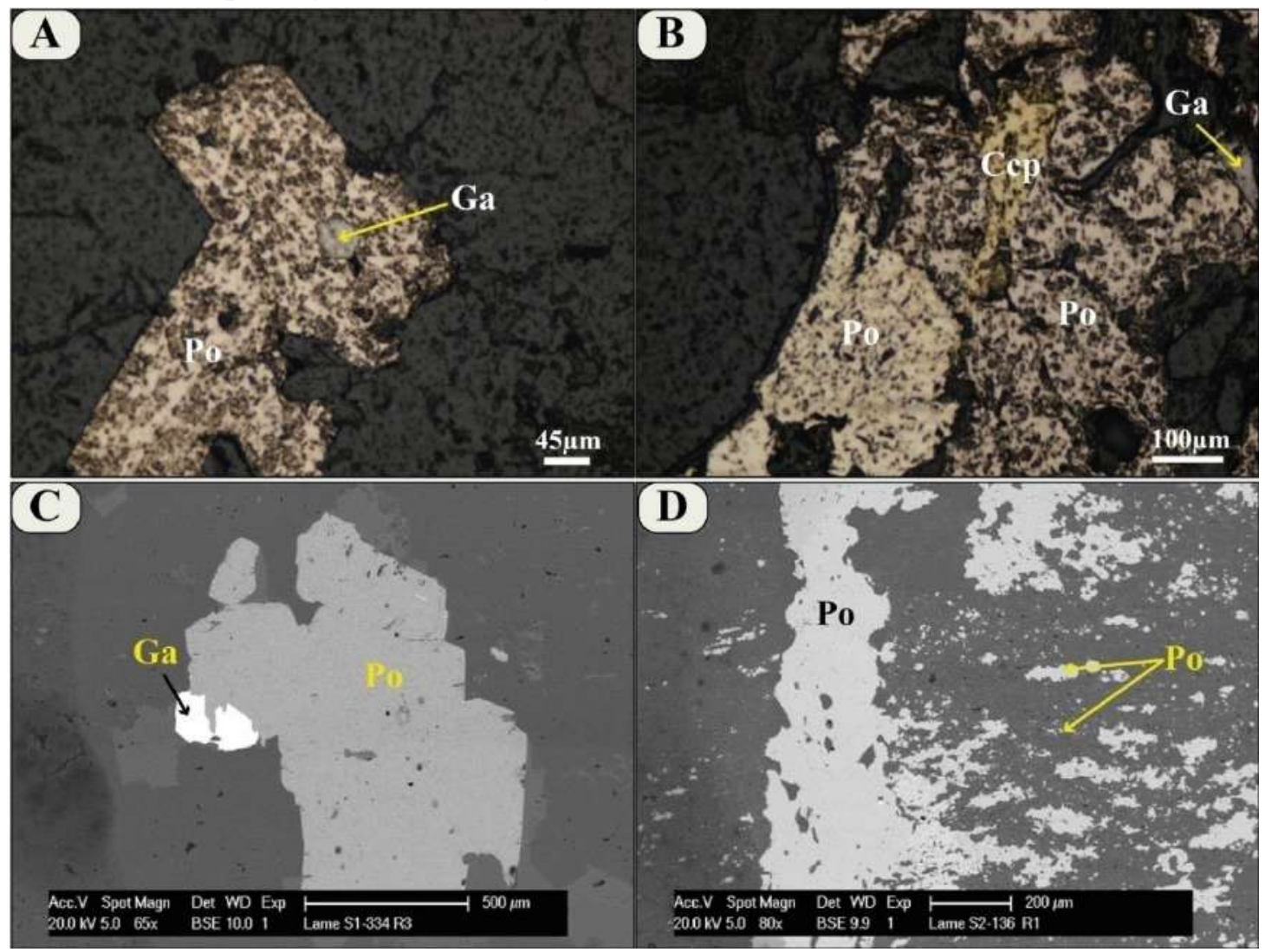

Figure 9 : Microphotographies illustrant l'abondance de la pyrrhotite dans les faciès fortement magnétique (Po: pyrrhotite, Ccp: chalcopyrite, Ga: galène).

- L'anomalie serait due à une nette différence entre la susceptibilité magnétique des différents faciès. Cette hypothèse mérite d'être 
élucidée par des mesures systématiques de la susceptibilité magnétique des différentes roches ;

- L'axe anomalique serait dû à un corps magnétique perturbateur non affleurant associé au volcanisme sous-marin. L'existence éventuelle de ce corps est quasiment impossible à prouver d'après les seules données de surface. Ceci nécessiterait l'application d'autres méthodes géophysiques tel que la gravimétrie et la polarisation provoquée et éventuellement des sondages. Cependant, la présence, à ce niveau, d'une minéralisation en dissémination et en veines à forte composante de pyrrhotite, pourrait pourrait etre l'expression en surface d'un amas de sulfures, massif pouvant être à l'origine de cette anomalie magnétique;

2-L'axe anomalique oriental coïncide avec l'affleurement de la zone de cisaillement orientale mettant en contact les deux principales unités lithologiques du secteur (les flyschs et les schistes gréseux). Il est situé à l'aplomb du dyke de gabbro à olivine riche en fentes de quartz. Mais légèrement décalé vers l'Est par rapport à son affleurement, qui par ailleurs, ne présente pas la même direction avec l'axe anomalique. Ces éléments ne permettent pas de voir une relation de cause à effet entre l'anomalie et les gabbros. Cependant, plusieurs hypothèses peuvent être énoncées pour expliquer les raisons possibles de cette anomalie :

- L'axe anomalique oriental coöncide avec la zone de cisaillement orientale; il serait dû à une grande différence de susceptibilité magnétique entre les formations mises en contact par le cisaillement. Mais dans ce cas, il est difficile d'expliquer la présence de cette anomalie uniquement dans la partie sud de la zone de cisaillement et son absence vers le nord malgré sa continuité dans cette direction ;

- La concentration de minéraux magnétiques (oxydes et sulfures de $\mathrm{Fe}$ ) qui pourraient être associés à d'autres substances métalliques $(\mathrm{Zn}, \mathrm{Cu}$, $\mathrm{Pb} . .$.$) et présenter éventuellement, dans ce cas, un intérêt économique.$

L'axe anomalique pourrait être lié à la circulation d'eau chargée en particules magnétiques le long de la zone de cisaillement. 


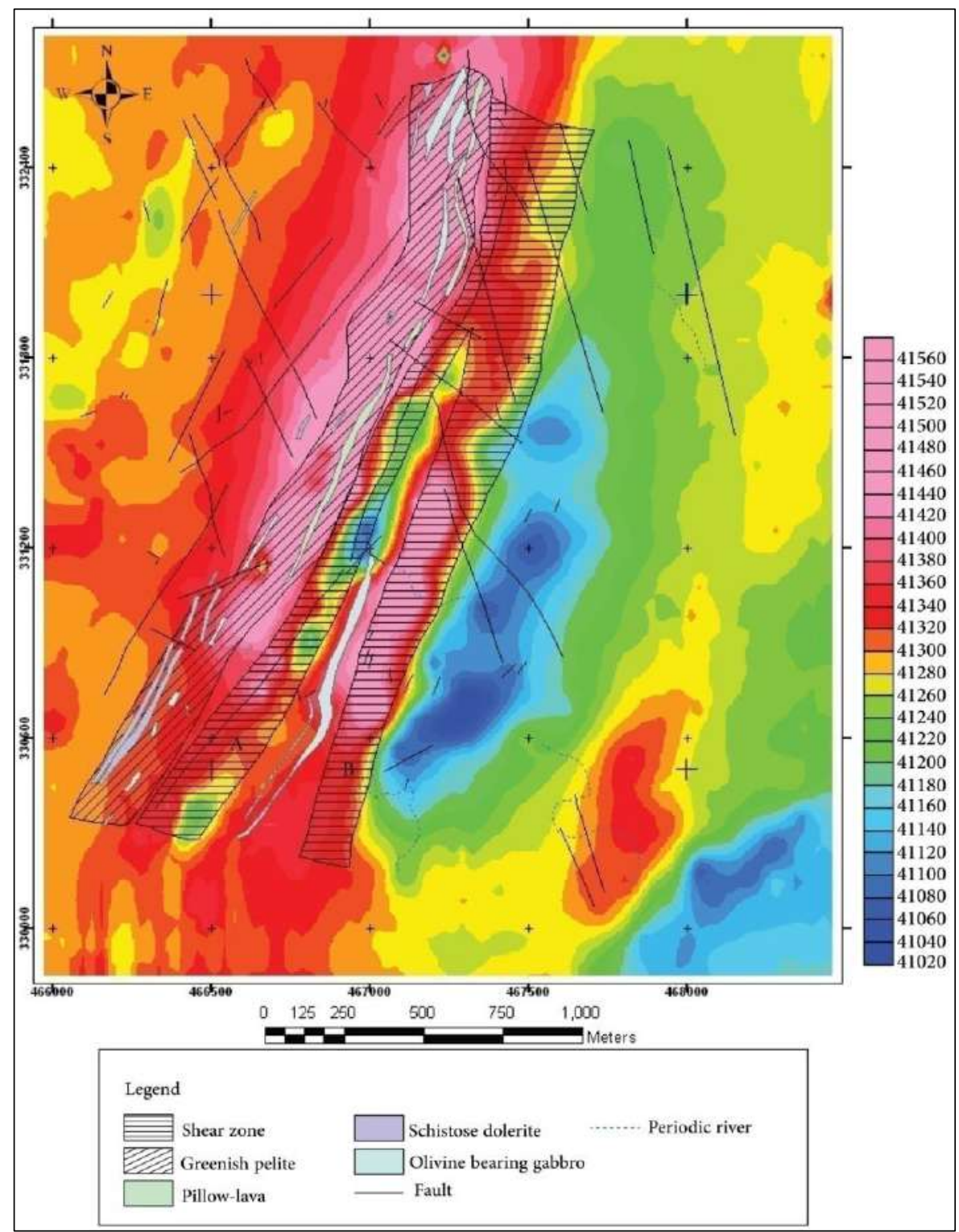

Figure 10 : carte de superposition du champ magnétique total et des données géologiques (zone de cisaillement, pélites verdâtres, roches basiques et les accidents).

Pour préciser la localisation des anomalies magnétiques, les coupes géologiques $\mathrm{AB}$ et $\mathrm{CD}$ ont été réalisé le long de deux profils magnétiques (voir fig. 3).

Les coupes géologiques $\mathrm{AB}$ et $\mathrm{CD}$, montrent deux anomalies importantes, dont la première, à l'ouest du profil, coïncide avec les roches basiques et les pélites verdâtres encaissantes, alors que la deuxième anomalie, plus à l'Est, coïncide avec la zone de cisaillement B (fig.11a et Fig.12). Les 
résultats de ces coupes sont en parfaite concordance avec les résultats de la superposition des données magnétiques et données géologiques.

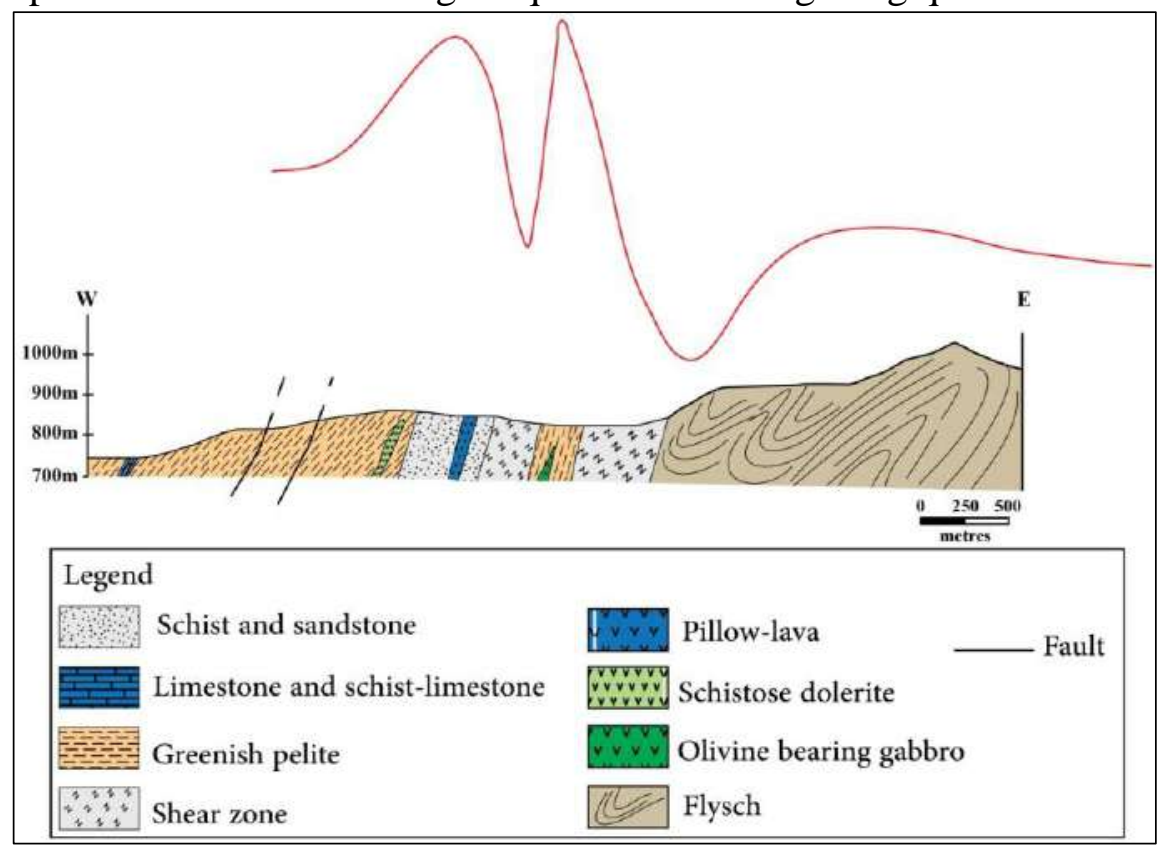

Figure 11 : coupe géologique $\mathrm{AB}$ le long de profil magnétique 300N

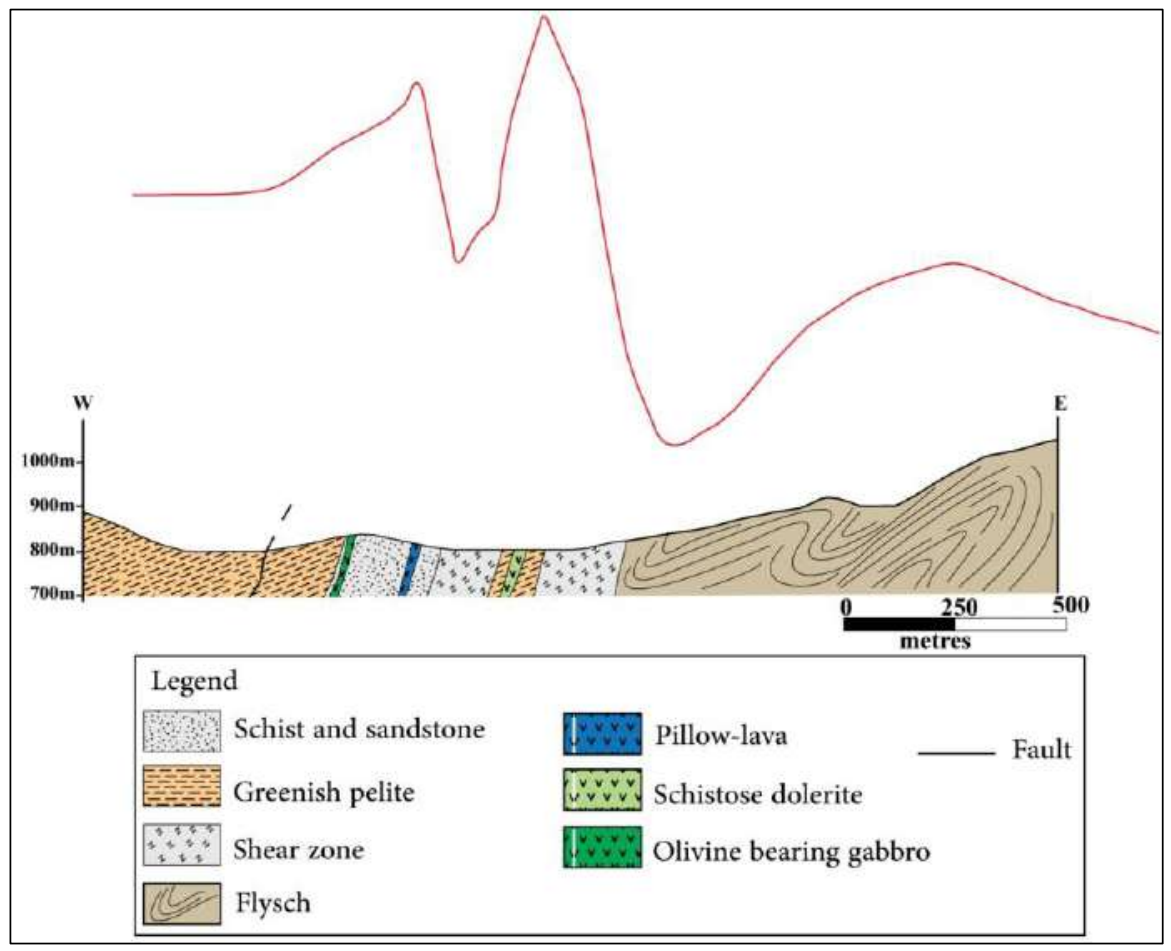

Figure 12 : coupe géologique CD le long de profil 100S 


\section{Conclusions}

De l'analyse et l'interprétation des données magnétiques du champ total de la région NNE d'Achemmach, se dégagent les résultats suivants :

La présence de deux axes anomaliques, l'un occidental et l'autre oriental, qui ont la même direction que les unités géologiques du secteur étudié :

- L'axe anomalique occidental coïncide avec l'affleurement des roches basiques et des pélites verdâtres encaissantes tronquées vers l'Est par la zone de cisaillement $\mathrm{A}$;

- L'axe anomalique oriental coïncide avec l'affleurement de la zone de cisaillement $\mathrm{B}$ mettant en contact les deux principales unités lithologiques du secteur (les flyschs et les schistes-grés).

Ces axes anomaliques traduisent soit la richesse des roches basiques (gabbros à olivine, pillow-lavas et dolérite), encaissées dans les pélites verdâtres, en minéraux magnétiques, tels que la magnétite et la pyrrhotite, soit la présence de corps magnétiques perturbateurs non affleurant à la surface, tels que des amas de sulfures à forte composante magnétique.

En se basant sur la forme des anomalies (en carte et en profils), il semblerait que des bandes, sous forme d'amas lenticulaires, riches en minéraux magnétiques (pyrrhotite) dans un encaissant faiblement magnétique (pélites verdâtres) en contact avec des roches non magnétiques vers l'est (flyschs), serait l'hypothèse la plus probable tenant compte à la fois des caractéristiques des anomalies magnétiques et données géologiques du secteur NNE d'Achmmach.

\section{References:}

1. Agard J. (1966). Données nouvelles sur le district de fluorine d'El Hammam-Berkamène (Maroc Central). Rapport service d'étude des gîtes minéraux, (843), Rabat.

2. Allary A., Andrieux J., Lavenu A. et al. (1972). Présence de décrochement dans la Méséta sud orientale (Maroc central). Comptes Rendus de l'Académie des Sciences,Paris, (274), série D, (pp. 653655).

3. Amenzou M. (1997). Typologie du zircon des granitoïdes hercyniens de la Meseta marocaine : zonation magmatique et implication géodynamique. Journal of AfricanEarth Sciences, Vol. (24), (1/2), (pp. 125-139).

4. Ben Abbou M. (1990). Evolution stratigraphique et structurale, au cours du paléozoïque, de la bordure nord du Massif central (région d'Agourai, Maroc). Thèse 3ème cycle, Fès, (pp. 214).

5. Ben Abbou M., Soula J. C., Brusset S. et al. (2001). Contrôle tectonique de la sédimentation dans le système de bassins d'avant-pays 
de la Meseta marocaine. Comptes Rendus de l'Académie des Sciences, Paris, série IIa (332), (pp. 703-709).

6. Berkhli M., \& Vachard D. (2002). Le Carbonifère du Maroc central : les formations de Migoumess, de Tirhela et d'Idmarrach. Lithologie, biostratigraphie et conséquences géodynamiques. Comptes Rendus des Géosciences, (334, pp. 67-72).

7. Bouabdelli M. (1989). Tectonique et sédimentation dans les bassins orogéniques: Le sillon viséen d'Azrou-Khénifra (Est du massif hercynien central). Thèse ès Sciences,Université Louis-Pasteur, Strasbourg, (pp. 257).

8. Boushaba A., Cailleux Y. (1992). Les relations métamorphismedéformation au voisinage des granitoïdes hercyniens du Maroc centrale. Bulletin de l'Institut Scientifique de Rabat, (16), (pp.15-22)

9. Cailleux Y. (1985). Les écailles anté-viséennes d'Ezzehiliga. Leur importance dans l'interprétation structurale du Maroc central. Comptes Rendus de l'Académie des Sciences, Paris, série II, (301), (pp. 497502).

10. Cattanéo G., Tahiri A., Zahraouiet al. (1993). La sédimentation récifale du Givétien dans la Meseta marocaine nord-occidentale. Comptes Rendus de l'Académie des Sciences, Paris. t. (317), série II, (pp. 73-80).

11. Cogney G., \&Faugères J.P. (1975). Précision sur la mise en place des épandages basaltiques de formations triasiques de la bordure septentrionale du Maroc central. Bulletin de la Société Géologique de France, (5), (pp. 721-733).

12. Dahmani A. (1985). Le métamorphisme dans l'auréole du granite d'Oulmès (Maroc central): étude pétrographique et relation avec les déformations hercyniennes. Thèse de 3ème cycle, Rabat, (pp. 150).

13. Diot H. (1989). Mise en place des granitoïdes hercyniens de la Méséta marocaine. Etude structurale des massifs de Sebt Brikine (Rehamna), de Zaer et d'Oulmès (Massif central) et d'Aouli Bou-Mia (Haute Moulouya). Implications géodynamiques. Thèse ès-sciences, Université Paul Sabatier Toulouse, France, (pp. 174).

14. El Hassani A. (1990). La bordure nord de la chaine hercynienne du Maroc. Chaîne "calédonienne" des Sehoul et plate-forme nordmésétienne. Thèse ès-Sciences, Université de Strasbourg, France, (pp. 208).

15. El Hassani A. (1990). La bordure nord de la chaine hercynienne du Maroc. Chaîne "calédonienne" des Sehoul et plate-forme nordmésétienne. Thèse ès- Sciences, Université de Strasbourg, France, (pp. 208). 
16. El Wartiti M. (1990). Le Permien du Maroc hercynien : étude géologique et implications paléogéographiques. Thèse de Doctorat d'Etat, Université Mohammed V,Rabat, (pp. 500).

17. Ettouhami M. (1992). Le Trias évaporitique du bassin de Khemisset (Maroc central): géométrie des dépôts, évolutions sédimentaires et géochimie. Thèse de Doctorat, Université Claude Bernard, Lyon I, (pp. 242).

18. Fadli D. (1991). Evolution sédimentaire et structurale des massifs des Mdakra et Khatouat : deux segmentshercyniens de la Méséta marocaine nord-occidentale.Thèse ès-Sciences, Rabat, (pp. 272).

19. Faik F. (1988). Le Paléozoïque de la région de Mrirt (Est du Maroc central). Evolution stratigraphique et structurale. Thèse de 3ème cycle, Université Paul Sabatier, Toulouse, (pp. 233).

20. FELENC J., ALJI M. BELLOT A., FOURNIER M., ET JIDORI S. (1989): Discovery of a concealdpyrhotite and base -metal bearing sulfide body et Hajar (Guemassa massif, Marocco), chron.Rech.Min. Special issue (pp. 15-19).

21. Gasquet D., Stussi J.M., \&Nachit H. (1996). Les granitoïdes hercyniens du Maroc dans le cadre de l'évolution géodynamique régionale. Bulletin Société géologique de France, (4), (pp. 517-528).

22. Giuliani G., Cheilletz A., \& Zimmerman J.L (1989). The emplacement, geochemistry and petrogenesis of two central Morocco Hercynian granites. Geotectonic implications. Journal of AfricanEarth Science, (9), (3-4), (pp. 617-629).

23. Habibi M. (1988). Le Paléozoïque de la région de Ain Leuh- Souq El Had (NE du Maroc central). Recherches stratigraphique et structurale. Thèse de 3ème cycle, Université de Toulouse, (pp. 186).

24. Habibi M. (1988). Le Paléozoïque de la région de Ain Leuh-Souq El $\mathrm{Had}$ (Ne du Maroc central). Recherches stratigraphique et structurale. Thèse de 3ème cycle,Université de Toulouse, (pp.186).

25. Hamoumi N. 1988. La plateforme ordovicienne du Maroc : dynamique des ensembles sédimentaires.Thèse es-sciences, Strasbourg, (pp. 239).

26. Hoepffner C. (1987). La tectonique hercynienne dans l'Est du Maroc. Thèse, université Louis-Pasteur, Strasbourg, (pp. 280).

27. Hoepffner C., Soulaimani A. \& Piqué A. (2005). The Moroccan Hercynides. Phanerozoic evolution of Africa. Journal of African Earth Sciences, (43), (pp. 144-165).

28. Huon S., Piqué A., \& Clauer N. (1987). Etude de l'orogenèse hercynienne au Maroc par la datation K/Ar de l'évolution métamorphique de schistes ardoisiers. Sciences Géologiques, Bulletin, Strasbourg, (40), (pp. 273- 284). 
29. Izart A., Chèvremont P., Tahiri A. et al. (2001). Carte géologique du Maroc au 1/50000, Feuille de Bouqachmir. Mémoire explicatif. Notes et Mémoire du service géologique du Maroc, ( $n^{\circ} 411$ bis), (pp. 60).

30. Jebrak M. (1984). Contribution à l'histoire naturelle desfilons F-Ba du domaine varisque : Essai de caractérisation structurale et géochimique des filons en extension et en décrochements. Massifs centraux français et marocains. Thèse de Doctorat d'Etat, Université d'Orléans, (pp. 470).

31. Kharbouch F. (1994). Les laves dévono-dinantiennes de la Meseta marocaine : étude pétrochimique et implications géodynamiques. Thèse d'état, Université de Brest, France (pp. 351).

32. Lagarde J. L. (1985). Cisaillements ductiles et plutons granitiques contemporains de la déformation hercynienne post-viséenne de la meseta marocaine. Hercynica, I, 1, (pp. 29-37).

33. Lakhloufi A. (1988). Etude structurale de la région de Brachwa, parties centrales et nord-orientale du bassin dévono-dinantien de Sidi Bettache (Maroc nordoccidental). Thèse de Doctorat, Ecole Nationale Supérieure, Rabat, (pp. 281).

34. Lazraq N. (1983). Contribution à l'étude micropaléontologique (principalement conodontes) du Dévonien de la région d'Oulmès (Maroc central). Thèse 3ème cycle, Paris VI, (pp. 95).

35. Mahmoudi A. \& Bertrand H. (2007). Geochemical identification of the central Atlantic magmatic province in folded domain, exemplified by the Moroccan Middle Atlas. Compte Rendu Géosciences, (V. 339), (pp. 545-552).

36. Mezougane.H et al. (2019), Communication orale: (Nouvelles données pétro-minéralogiques et géochimiques des formations magmatiques basiques du secteur NNE Achemmach (Maroc central). Colloque International 3MA- 11;23-24 Avril 2019 à El Jadida, Maroc.

37. Michard A. (1976). Elément de géologie Marocaine. Notes et mémoires du service géologique, Rabat., Maroc, (n²52), (pp. 408).

38. Mrini Z., Rafi A., Duthou J-L. et al. (1992). Chronologie Rb-Sr des granitoïdes hercyniens du Maroc: conséquences. Bulletin de la Société Géologique de France, (163, pp. 281-291).

39. Ntarmouchant A. (2003). Le magmatisme associé à l'orogenèse du Maroc varisque: exemple du magmatisme du bassin méridional d'Azrou- Khénifra (Est du Maroc hercynien central). Thèse d'Etat, Université Sidi Mohammed Ben Abdellah, Fès, 309p.

40. Ouabid M., Ouali H., Garrido C. J., Acosta-Vigil A., Román-Alpisteb M.J., Dautria J-M., Marchesi C., Hidas K. (2017). Neoproterozoic granitoids in the basement of the MoroccanCentral Meseta: 
Correlation with the Anti-Atlas at the NW paleo-margin of Gondwana. Precambrian Research, (299), (pp. 34-57).

41. Oubbih J. (1991). Le Maroc central méridional (région de Moulay Bou Azza) : Stratigraphie du Paléozoïque et tectonique hercynienne. Thèse de 3ème cycle, Université Mohamed V, Rabat, (pp. 176).

42. Piqué A. \& Michard A. (1981). Les zones structurales du Maroc hercynien. Bulletin de la Société Géologique de France, Strasbourg, (34), (pp. 135-146).

43. Piqué A. \& Michard A. (1981). Les zones structurales du Maroc hercynien. Bulletin de la Société Géologique de France, Strasbourg, (34), (pp. 135-146).

44. Piqué A. \& Michard A. 1981. Les zones structurales duMaroc hercynien. Bulletin de la Société Géologique de France, Strasbourg, (34), (pp. 135-146).

45. Piqué A. (1994). Géologie du Maroc. Les domaines régionaux et leur évolution structurale. Edition Pumag, Marrakech, (pp. 284).

46. Remmal T., Ben Abbou M., Youbi N. et al. (1999a). Le bassin permien de Bou Achouch: une structure sur décrochement NE-SW senestre associée a un volcanisme calco-alcalin d'intraplaque. Société Géologique du Nord, T.7 (2ème série), (pp. 135-146).

47. Roddaz M, Brusset S; Soula J-C, Debat P, Ben Abbou M., Beziat D, Driouch Y, Christophoul F, N'Tarmouchant A., \&Deramond J. (2002) -Foreland basin magmatism in the Western Moroccan Meseta and geodynamic inferences, Tectonics, (21: pp. 181-198).

48. Saidi A. (2005). Etat de contrainte et mécanismes d'ouverture et de fermeture des bassins permiens de la Meseta marocaine. Apport de la Télédétection à la reconnaissance des faciès et des réseaux de failles. Thèse de Doctorat, Université Mohammed V, Rabat, (pp. 222).

49. Saidi A., Tahiri A., Ait Brahim L. et al. (2002). Etats de contraintes et mécanismes d'ouverture et de fermeture des bassins permiens du Maroc hercynien. L'exemple des bassins des Jebilet et des Réhamna. Comptes Rendus Géosciences, Paris, (334), (pp. 221-226).

50. Sonnet Ph. (1981). Les skarns à Sn, W, B de la région d'El Hammam (Maroc central). Thèse de Doctorat, Faculté des Sciences Appliquées, Université Catholique de Louvain, Belgium, (pp. 512).

51. Tahiri A. \&Hoepffner C. (1987). La faille d'Oulmès:cisaillement ductile et tectonique tangentielle, Maroc central hercynien. Bulletin de l'Institut Scientifique, Rabat, (11), (pp. 59-68).

52. Tahiri A. (1991). Le Maroc central septentrional : stratigraphie, sédimentologie et tectonique du Paléozoïque ; un exemple de passage des zones internes aux zones externes de la chaîne hercynienne du 
Maroc. Thèse ès-Sciences, Université de Bretagne occidentale, Brest, (pp. 300).

53. Tahiri A. (1994). Tectonique hercynienne de l'anticlinorium de Khouribga-Oulmès et du synclinorium de Fourhal. Bulletin de l'Institut Scientifique, Rabat, (18), (125-144)., Tahiri A. \&Hoepffner C. (1987). La faille d'Oulmès: cisaillement ductile et tectonique tangentielle, Maroc central hercynien. Bulletin de l'Institut Scientifique, Rabat, (11), pp. 59-68).

54. Tahiri A., Montero P., El Hadi H., Poyatos D., Azor A., Bea F., Simancas J.F., González Lodeiro F. (2010) - Geochronological data on the Rabat-Tiflet granitoids: Their bearing on the tectonics of the Moroccan Variscides, Journal of African Earth Science, (57), (pp.113).

55. Termier H. (1936). Etude géologique sur le Maroc central et le Moyen Atlas septentrional. Notes et Mémoires du Service de Géologie du Maroc, (33), (pp. 1566).

56. Zahraoui M. (1994). Le Dévonien inferieur et moyen de la meseta nord occidentale. Bulletin de l'Institut Scientifique, Rabat, (18), (pp. 43-56).

57. Zouine M. (1986). Evolution structurale tardi-hercynienne de la bordure NW du Maroc central entre Tiddas et JbelTariona. Thèse de Doctorat, Ecole Nationale Supérieure, Rabat, (pp. 131). 